\title{
Physical fitness changes among amateur soccer players: effects of the pre-season period
}

\author{
Rui Silva ${ }^{1}$, Ricardo Lima $^{1,2}$, Miguel Camões ${ }^{1,2}$, César Leão $^{1}$, Sérgio Matos ${ }^{1}$, Joel Pereira ${ }^{1}$, Pedro \\ Bezerra $^{1,2}$, Filipe Manuel Clemente ${ }^{1,3}$
}

${ }^{1}$ Escola Superior Desporto e Lazer, Instituto Politécnico de Viana do Castelo, Rua Escola Industrial e Comercial de Nun'Álvares, 4900-347, Viana do Castelo, Portugal; 2 The Research Centre in Sports Sciences, Health Sciences and Human Development, Vila Real 5001-801, Portugal; ${ }^{3}$ Instituto de Telecomunicações, Delegação da Covilhã, Lisbon 1049-001, Portugal

\begin{abstract}
Study aim: To assess changes in physical fitness of amateur soccer players after a pre-season training period and baseline fitness dependencies.

Material and methods: Twenty-one amateur soccer players were assessed during the pre-season. The following physical variables were assessed before and after a two-month pre-season training period: (i) cardiorespiratory fitness, (ii) strength and power, and (iii) change of direction (COD).

Results: Significant decreases were found for countermovement jump (CMJ) $(p<0.001 ; d=1.161)$, drop jump (DJ) (p = 0.014; $\mathrm{d}=0.958)$, and horizontal jump (HJ) ( $\mathrm{p}=0.042 ; \mathrm{d}=0.640)$, while no significant changes were found for the overall variables from the beginning to the end of pre-season. Fit players revealed significant decreases for CMJ $(p=0.002 ; d=-2.495)$, DJ $(\mathrm{p}=0.004 ; \mathrm{d}=-1.760), \mathrm{HJ}(\mathrm{p}=0.028 ; \mathrm{d}=-1.005)$, COD deficit $(\mathrm{p}=0.034 ; \mathrm{d}=1.013)$, and maximal aerobic speed (MAS) $(\mathrm{p}=0.026 ; \mathrm{d}=-4.053)$. No significant changes were found for unfit players.

Conclusions: Amateur soccer coaches should consider assessing physical qualities at the beginning of pre-season and use the free-of-charge monitoring tools such as session-rate of perceived exertion (s-RPE) during the training process.
\end{abstract}

Keywords: Soccer - Performance - Sports training - Physical fitness - Pre-season

\section{Introduction}

Soccer is characterized by its intermittent nature, in which players are expected to perform mainly low-intensity running activities interspersed with high-intensity explosive actions during both training and competition [2]. This requires that soccer players maintain adequate aerobic and anaerobic capacity. Soccer players have shown average of $\mathrm{VO}_{2 \max }$ values between 51 and $62.9 \mathrm{ml} \cdot \mathrm{kg}^{-1} \cdot \mathrm{min}^{-1}$ [5], and maximal aerobic speed ranging between approximately 13 and $17 \mathrm{~km} / \mathrm{h}$ [3]. Considering the high-intensity actions, players are required to perform sprints, jumps, changes-of-direction, accelerations and decelerations during training and competition, which are associated with the athletes' strength and power capacity [39].

On one hand, during training, adult elite soccer players can cover total distances that reach approximately $7000 \mathrm{~m}$ during a training/practice session, of which $990 \mathrm{~m}$ are covered at high speeds and approximately 337 to $444 \mathrm{~m}$ are covered at sprint speeds [8]. Up to $178 \mathrm{~m}$ can be covered while accelerating, and up to $162 \mathrm{~m}$ can be covered while decelerating [13]. On the other hand, during a match, elite players can perform a total of approximately 15 header jumps and can cover a total distance of about $10 \mathrm{~km}$ to $13 \mathrm{~km}$ [2]. Meanwhile, amateur players tend to cover about $5 \%$ less distance. However, elite players cover about $2.43 \mathrm{~km}$ while running at a high intensity and $0.65 \mathrm{~km}$ at a sprint, with amateur players covering $28 \%$ and $58 \%$ less, respectively, at these speeds [23]. Also, recent research has revealed the importance of strength in the overall soccer training process, which seems to be associated with greater physical fitness development [26, 32]. As mentioned above, soccer players need high levels of aerobic capacity to sustain the more than $70 \%$ of lower-intensity actions during a match [2]. Also, they need high levels of 
strength, which is related to acceleration and sprint ability, as well as power-related tasks such as vertical jumps that are required for disputes in the air and scoring header goals [37, 39].

Based on the training loads and other multifactorial life stressors, variations throughout the season should be addressed with regard to players' body composition, level of physical fitness, and motor skills [22]. The season can be organized into three different phases with different types of stimuli, and it would be convenient to expect changes in the aforementioned variables as a result [2]. For instance, the aerobic power and the time to exhaustion seem to increase throughout the first phase of the season. This is typically followed by a maintenance phase through to the end of the season [22]. Also, there seem to be differences in squat jump (SJ) and countermovement jump (CMJ) performance between the pre-season and the middle of the season. Specifically, a $10.1 \%$ increase in the height reached in the SJ and a $9.8 \%$ increase for the CMJ have been reported. Furthermore, a 13.7\% increase in 1 repetition maximum (1RM) for the deadlift during the pre-season has been observed, and this increased to $6 \%$ during the mid-season [15]. It has been reported that strength training of the upper body improves $\mathrm{VO}_{2 \max }$ by $2.6 \%$ and of upper body maximal strength by $30 \%$, resulting in gains related to the ability to react faster and engage in body contact with opponents [31]. The changes observed in physical fitness in adult athletes have been well documented. In fact, improvements in aerobic and anerobic fitness were shown after the pre-season period, in a study conducted on 23 male soccer players from a first league Israeli team, in which the authors associated those improvements with the type of training implemented during that particular period of the season [21].

Soccer players have demonstrated reasonable levels of physical fitness, with high values of $\mathrm{VO}_{2 \max }$. [7]. However, these values can vary according to the different tactical positions of the players. Midfielders have the highest $\mathrm{VO}_{2 \max }$ values, followed by defenders, forwards, and, finally, goalkeepers [33]. At the beginning of the pre-season, the athletes have a relatively low level of fitness due to the off-season period, presenting lower $\mathrm{VO}_{2 \max }$ values, although improvements of $7 \%$ versus the post-preseason are achieved and only $2 \%$ of improvements remain until the mid-season, and $\mathrm{VO}_{2 \max }$ is then maintained for the rest of the season [21].

The changes observed during the season seem to depend on the values observed at the beginning of the preseason, which may be related to the low values that the players present at the end of the off-season. In fact, a recent study conducted on 130 elite Spanish soccer players showed that the assumption that at the end of a pre-season period an improvement in aerobic fitness is expected may not be so straightforward as the changes observed after the pre-season period tend to vary significantly between seasons [18]. Thus, the effect of training stimuli significantly increases these values until a plateau is reached [22]. In addition, the improvements in $\mathrm{VO}_{2 \max }$ allow athletes to cover $20 \%$ greater distances at high intensities (i.e., at $>90 \%$ of their maximal heart rate). Positive changes have also been found in terms of athletes' physical fitness during the three different periods of the season (off-season, pre-season and in-season). The time spent in the 40-m and 20 -m sprint decreases by $2 \%$ from the pre-season to the off-season. This decrease could be related to the soccer conditioning and might be subject to variations in the different periods of the season [14].

Furthermore, a decrease in the time of execution of the pro-agility test of approximately $3 \%$ has been observed from the pre-season to the post-season. Also, decreases of $3 \%, 4 \%$, and $1 \%$ have been reported for the $10-\mathrm{m}$, 30-m, and 40-m sprint, respectively, from the pre-season to the post-season [19]. To assess the maximum strength of soccer athletes, the isometric mid-thigh pull (IMTP) test has been used, and it seems to be correlated with movements that are typical of good performance in soccer players, such as sprint speed and vertical jump height [36]. For instance, an investigation aimed to assess the relationship between strength, sprint speed, agility, and IMTP test performance in rugby athletes. Positive correlations were observed between the IMTP and 1RM squat, the time to complete a 5-m sprint, and the time taken in the pro-agility test [36], which is perceived as a viable test for measuring the motor skills that are inherent in soccer performance.

Anthropometric, physical fitness, and motor skill changes are well documented in elite soccer players [17, 20]. However, there is a lack of evidence about the influence of the pre-season period on changes in the fitness status of amateur players. Evidence is also scarce concerning the role of baseline values in such changes. The evidence above demonstrates the importance of an adequate understanding of the physical demands of soccer and their changes even in amateur competitions. In addition, it is essential to collect data on the athletes' initial state at the beginning of the season, to track positive or negative changes during the training process. This provides amateur soccer coaches and athletes with the tools necessary for better training planning and to adjust training accordingly.

For those reasons, the first purpose of this study was to investigate changes in fitness levels after a pre-season training period; and the second purpose was to describe amateur players' physical changes in relation to their baseline fitness levels. We hypothesize that physical fitness will improve after a training period in the different outcomes. 


\section{Material and methods}

\section{Participants}

The sample consisted of 21 amateur soccer players (age: $23.6 \pm 7.3$ years old; body height: $178.2 \pm 0.1 \mathrm{~cm}$; body mass: $75.2 \pm 9.2 \mathrm{~kg}$ in the first assessment and $76.0 \pm 9.2 \mathrm{~kg}$ in the second assessment; fat mass percentage: $11.5 \pm 2.0 \%$ in the first assessment and $10.9 \pm 1.7 \%$ in the second assessment). All players were from the same team and were competing in the 2019/2020 season of the first division of the Portuguese Amateur Championship.

The participants comprised two goalkeepers, four central defenders, one external defender, six midfielders, five wingers, and two forwards. Players were assessed at two different times: (i) at the beginning of the pre-season phase (July 2019) and (ii) two months later (i.e., at the end of the pre-season) (September 2019). During the pre-season period, players trained four times a week ( $\sim 90$ minutes/ session) and participated in one friendly match each week. The inclusion criteria were: (i) participation in both assessments and (ii) not being injured throughout the period of assessment and between assessments for more than one week. Before being assessed, the player was informed of the study's protocol and procedures. After their voluntary agreement, they signed a free informed consent form. The study followed the ethical standards of the Declaration of Helsinki for research in humans. The study was approved by the local scientific committee with the code number ESDL.2019.07.001.

\section{Study design}

This study used a within-subjects observational cohort design to examine changes in participants' aerobic and anaerobic performance via physical performance tests.

Anthropometric measures (body mass, height, fat mass percentage), cardiorespiratory fitness (Bronco test, maximal aerobic speed), strength and power (isometric mid-thigh pull, squat jump, countermovement jump, drop jump, horizontal jump), and agility and speed (pro-agility) were assessed twice - once before the beginning of the pre-season training phase (July 2019) and again at the end of the pre-season period (September 2019). After the first assessment (baseline), the median values for each fitness test were determined. Based on these values, players within the upper limit (better than the median) were classified as fit players, and the other players were classified as unfit players. This classification was done for each test; thus, a player may have been classified as fit in some tests and unfit in others. We decided to analyze the changes between the baseline and the post-training assessments for fit and unfit players in order to verify whether baseline levels influenced improvements or declines in players' performances.
Both assessments took place under similar conditions. Both were done 48 hours after the last training session/ match, on the same day of the week (Monday) at the same time (5:00 p.m.) on natural turf and were carried out by the same trained sports science technicians.

\section{Anthropometrics and body composition}

Body weight was measured to the nearest $0.1 \mathrm{~kg}$ using a Tanita BC-601 instrument, and height was measured using a stadiometer to the nearest $0.1 \mathrm{~cm}$ (Seca 217, Hamburg, Germany). Skinfold thickness was measured at eight sites (triceps, biceps, subscapular, suprailiac, supraspinal, abdominal, thigh, and calf) using a skinfold caliper (Harpenden, West Sussex, UK). Girth was measured at four sites (arm relaxed, arm flexed, thigh, and calf) using a Cescorf flexible anthropometric tape. Body fat percentage was calculated using the formula recommended by Reilly [29].

All measurements were performed by the same experienced and certified observer who had a level 2 certification from the International Society for the Advancement of Kinanthropometry (ISAK), using the standardized techniques of the ISAK.

\section{Cardiorespiratory fitness}

To assess cardiorespiratory fitness, all players performed the 1200-m shuttle run test (Figure 1) (also known as the Bronco test), as previously described by Kelly and Wood [16]. Players had to perform five repetitions as fast as they could, starting at a baseline cone and then running forward to the next cone $20 \mathrm{~m}$ away. They then returned to the baseline and performed another forward run to a cone $40 \mathrm{~m}$ away. They returned to the baseline once again before making a final forward run to a cone $60 \mathrm{~m}$ away and finishing the test by returning to the baseline. Players were familiarized with the test before performing it, thus increasing the accuracy of their pace during the test.

To determine the lowest speed at which $\mathrm{VO}_{2 \max }$ occurred (e.g., the maximal aerobic speed (MAS)), the total time, in seconds, taken to complete the test was recorded. MAS was then calculated by dividing the 1200 -m distance by the recorded time. However, the time recorded for each player was corrected according to the following equation: MAS $=1200 /($ time in seconds -20.3$)[1]$.

\section{Strength and power}

For the assessment of strength and power, the isometric mid-thigh pull test (IMTP), squat jump (SJ), countermovement jump (CMJ), drop jump (DJ), and horizontal jump (HJ) tests were conducted on all players. A description and demonstration of the procedures were given before the test commenced.

For the IMTP test, a single ratchet strap was placed around a weight plate and attached to a crane scale that 


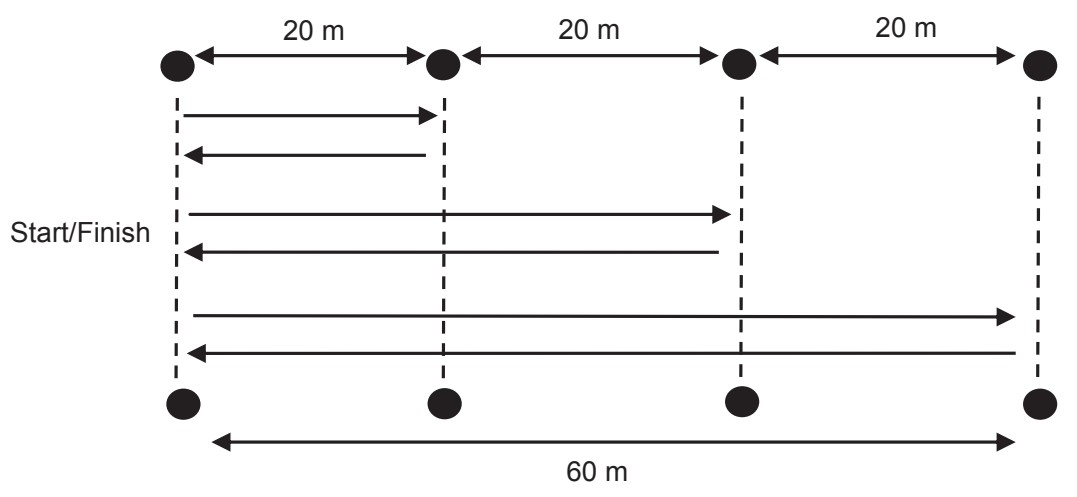

Figure 1. 1200-m shuttle (The Bronco) test configuration

was already attached to a bar. The players were instructed to adjust the position of the bar to a comfortable (midthigh) position by shortening or lengthening the ratchet straps before performing the pull. Then, the players were instructed to pull the bar with the maximum strength they could and to maintain the pull for three seconds. They were then given five seconds to rest. This process was then repeated twice. The peak force produced during the test was registered by recording all the trials on video and later analyzing them in slow motion. All measurements were recorded in $\mathrm{kg}$ but were later converted into newtons $(\mathrm{N})$ by calculating the peak force in $\mathrm{kg}$ multiplied by gravity. The average of peak force, both in $\mathrm{kg}$ and $\mathrm{N}$, for each of the three trials was calculated. The relative force was also recorded by dividing the peak force, in N, by the player's body mass. The IMTP test using a crane scale has been documented and proven to be a valid and reliable method that is not significantly different from the force plates method [35].

The vertical SJ, CMJ, and DJ tests were performed on a DIN-A2 contact platform (Chronojump, Spain) connected to a Chronopic 3 microcontroller. This device was connected to a portable computer with Chronojump free software installed so that it could analyze the received information. The Chronojump contact platform, microcontroller, and software used are known to be valid and reliable tools for measuring vertical jump times. For all frequencies at which the microcontroller was tested, the mean absolute errors were $0.13 \%$ and $0.14 \%$ for contact time and in-flight time, respectively. Also, a difference of only $1.40 \pm 0.92 \%$ was found, and an intraclass correlation coefficient (ICC) of 0.95 was observed between the DIN-A2 contact platform and the Ergojump-Boscosystem platform reference measure [4].

All players were told to stand on top of the contact platform and perform a practice jump for each jump test. Then, three trials were performed; each player's best score of the three vertical jump tests was recorded. For the SJ test, players were instructed to place their hands on their hips during the full movement to prevent arm swinging, and they were to start the movement from a knee flexion position that they were to hold for three seconds before jumping as high as possible and landing in the same place on the contact platform.

For the CMJ test, players started from a standing position with both hands on their hips and allowed their comfortable depth of knee flexion in the descending phase of the jump to be determined. During the flight, players had to maintain hip and knee extension while jumping as high as possible and landing in the same place and with both feet at the same time without removing their arms from their hips.

For the assessment of reactive jump capacity, the DJ test was performed. Players started from a standing position on top of a 30-cm-high box with both hands on their hips. When the players were instructed to start the test, they initiated the movement by stepping a single leg out of the box and then dropping their body on the force plate. Then, as the players reached the ground, they started the vertical take-off as fast as possible with their knee and hip joints extended. This was followed by the second landing, for which players were instructed to land with their weight correctly distributed and to assume a half-squat position to absorb the impact of the jump.

For the assessment of the horizontal jump, players started from a standing position and were allowed to swing their arms and bend their knees in a comfortable position. They were then to jump as far as possible without losing their balance when landing with both feet simultaneously. A loss of balance resulted in a null attempt, meaning that the player needed to repeat the trial. A metric measuring tape was attached to the ground to measure the jump length, and a take-off line was placed on the ground using tape. The measurement was taken at the back of whichever heel was the furthest back. All horizontal jumps were repeated three times, and the best score was recorded. 


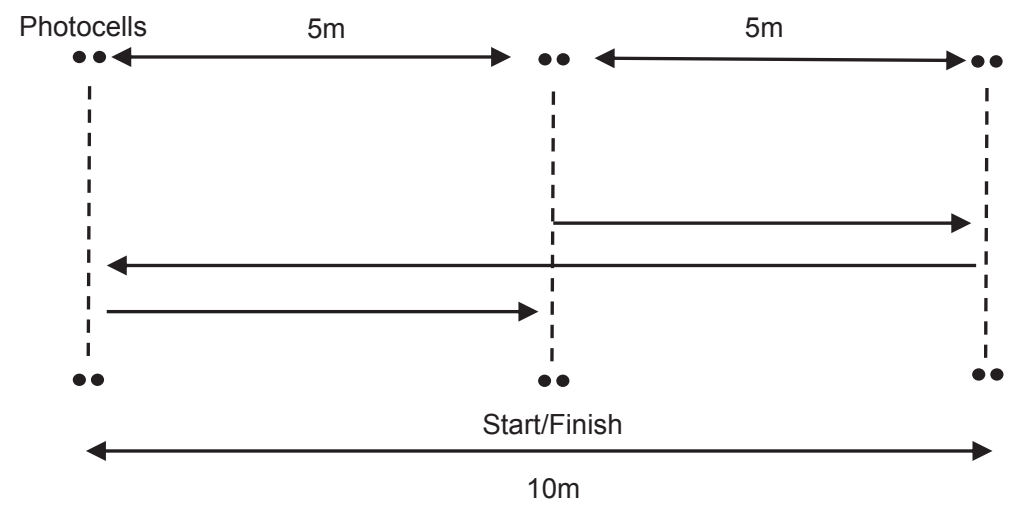

Figure 2. Pro-agility test configuration

\section{Change of direction and speed}

For the assessment of speed and change of direction (COD), the pro-agility test (Figure 2) was conducted as previously described [34], in which three pairs of photocells (Witty Gate, Microgate, Italy) were placed at distances of 0 $\mathrm{m}$ (starting point), $5 \mathrm{~m}$ to the right, and $5 \mathrm{~m}$ to the left. The players started from a three-point stance at the $0 \mathrm{~m}$ starting point. The first 5-m run was taken in the direction of the hand that was in contact with the ground. When the players reached the first 5-m external line, they touched the line with their lead foot and hand. They then ran forward as fast as possible to the opposite 10-m external line, again touching it with their lead foot and hand when they reached it. Finally, they ran forward to the 0 -m starting point, completing the test when they crossed the starting line. All players were instructed to perform the test three times on each side; trials were separated by two minutes of rest.

For the purpose of distinguishing COD ability from linear speed ability, the COD deficit (previously recommended by Nimphius [25]) was calculated as the difference between the first $10-\mathrm{m}$ sprint split time during the 20-m pro-agility test.

\section{Statistical procedures}

The results were expressed as mean (standard deviation). After confirmation of normality $(p>0.05)$ and homogeneity $(p>0.05)$ assumptions, a paired sample t-test was used to determine the differences between the pre and post assessments, followed by Cohen's $d$ to analyze the effect size (ES). The following classification to measure the magnitude of ES was used: $\leq 0.2$, from 0.3 to 0.6 , from 0.6 to 1.2 , from 1.2 to 2.0 and $>2.0$ were considered trivial, small, moderate, large and very large, respectively. An independent sample t-test was used to determine the differences between the baseline and post assessments for fit and non-fit players. All statistical analyses were performed using SPSS statistical analysis software (SPSS version 24.0, IBM corp., Chicago, USA). The level of statistical significance was set at $\mathrm{p}<0.05$.

\section{Results}

Table 1 presents a descriptive analysis of the different fitness variables in the pre and post pre-season periods. Significant decreases in performance were found from the baseline to the post period assessment for countermovement jump (mean percentage change [dif]: $-13.7 \%$; $\mathrm{p}<0.001 ; \mathrm{d}=1.161$, moderate effect size), for drop jump (dif: $-10.0 \% ; \mathrm{p}=0.014 ; \mathrm{d}=0.958$, moderate effect size) and for horizontal jump (dif: $-3.0 \% ; \mathrm{p}=0.042 ; \mathrm{d}=0.640$, moderate effect size).

A comparison between the baseline values (first assessment) and the post values of the players categorized as fit was made and can be found in Table 2. Significant differences for fit players were found between the baseline and post assessments for countermovement jump (dif: $-16.8 \% ; \mathrm{p}=0.002 ; \mathrm{d}=-2.495$, very large effect size), drop jump (dif: $-15.5 \% ; \mathrm{p}=0.004 ; \mathrm{d}=-1.760$, large effect size), horizontal jump (dif: $-3.2 \% ; p=0.028 ; d=-1.005$, large effect size), and for change of direction deficit (dif: $413 \% ; p=0.034 ; d=1.013$, moderate effect size). Also, a significant difference was found for maximal aerobic speed (dif: $-16.5 \% ; p=0.026 ; d=-4.053$, very large effect size).

A comparison between the baseline values (first assessment) and the post values of the players categorized as unfit was made and can be found in Table 3. No significant differences for unfit players were found.

\section{Discussion}

The aims of the present study were to investigate the changes in fitness levels after a pre-season training period, and to describe amateur players' physical changes, considering their baseline fitness levels. The results revealed significant changes only in the jump performance assessments after the pre-season training period. The level of 
Table 1. Physical fitness changes in pre-season training period

\begin{tabular}{lccccc}
\hline Fitness determinants & Pre $($ mean $\pm \mathrm{SD})$ & Post $($ mean $\pm \mathrm{SD})$ & $\%$ change $\pm \mathrm{SD}($ Post-Pre $)$ & $\mathrm{p}$ & $\mathrm{d}$ \\
\hline IMTP Peak $[\mathrm{kg}]$ & $128.42 \pm 26.11$ & $127.14 \pm 27.19$ & $-1.5 \pm 5.5$ & 0.747 & 0.048 trivial \\
IMTP Avg $[\mathrm{kg}]$ & $119.07 \pm 24.92$ & $120.07 \pm 26.35$ & $0.5 \pm 5.1$ & 0.763 & -0.039 trivial \\
Relative Force $[\mathrm{N} / \mathrm{kg}]$ & $16.57 \pm 4.05$ & $16.43 \pm 3.25$ & $-0.6 \pm 7.1$ & 0.834 & 0.038 trivial \\
CMJ $[\mathrm{cm}]$ & $35.71 \pm 4.20$ & $30.93 \pm 4.03$ & $-13.7 \pm 4.8$ & 0.000 & 1.161 moderate \\
DJ $[\mathrm{cm}]$ & $31.07 \pm 4.29$ & $27.71 \pm 2.49$ & $-10.0 \pm 6.1$ & 0.014 & 0.958 moderate \\
HJ $[\mathrm{cm}]$ & $227.64 \pm 10.69$ & $220.71 \pm 10.95$ & $-3.0 \pm 2.5$ & 0.042 & 0.640 moderate \\
ProAgility [s] & $5.14 \pm 0.22$ & $5.07 \pm 0.27$ & $-1.4 \pm 2.0$ & 0.279 & 0.284 small \\
COD Deficit $[\mathrm{s}]$ & $0.07 \pm 0.33$ & $0.22 \pm 0.10$ & & 0.138 & -0.615 moderate \\
Bronco $[\mathrm{s}]$ & $305.71 \pm 38.82$ & $320.43 \pm 37.10$ & $4.9 \pm 6.8$ & 0.260 & -0.387 small \\
MAS [m $\cdot \mathrm{s}^{-1}$ ] & $3.95 \pm 0.46$ & $3.78 \pm 0.40$ & $-4.6 \pm 6.7$ & 0.234 & 0.394 small \\
\hline
\end{tabular}

d: standardized effect size of Cohen; IMTP Avg = Average Isometric mid-thigh pull; CMJ = countermovement jump; DJ = drop jump; HJ = horizontal jump; $\mathrm{COD}=$ change of direction; MAS = maximal aerobic speed.

Table 2. Fit player changes between baseline and post assessments

\begin{tabular}{lccccc}
\hline Fitness determinants & Pre $($ mean $\pm \mathrm{SD})$ & Post $($ mean $\pm \mathrm{SD})$ & $\%$ change $\pm \mathrm{SD}$ (Post-Pre) & $\mathrm{p}$ & $\mathrm{d} \pm \mathrm{SD}$ \\
\hline IMTP Peak $[\mathrm{kg}]$ & $149.11 \pm 18.46$ & $144.9 \pm 16.16$ & $-2.7 \pm 7.1$ & 0.477 & $-0.219 \pm 0.58$ small \\
IMTP Avg $[\mathrm{kg}]$ & $135.99 \pm 17.35$ & $137.60 \pm 14.02$ & $1.4 \pm 6.5$ & 0.726 & $0.108 \pm 0.50$ trivial \\
Relative Force $[\mathrm{N} / \mathrm{kg}]$ & $18.59 \pm 3.40$ & $17.53 \pm 3.66$ & $-6.2 \pm 6.0$ & 0.119 & $-0.352 \pm 0.35$ small \\
CMJ $[\mathrm{cm}])$ & $38.70 \pm 2.49$ & $32.38 \pm 4.08$ & $-16.8 \pm 6.1$ & 0.002 & $-2.495 \pm 0.99$ very large \\
DJ $[\mathrm{cm}]$ & $33.17 \pm 3.25$ & $28.04 \pm 2.82$ & $-15.5 \pm 6.7$ & 0.004 & $-1.760 \pm 0.83$ large \\
HJ $[\mathrm{cm}]$ & $235.33 \pm 4.72$ & $228.00 \pm 10,08$ & $-3.2 \pm 2.1$ & 0.028 & $-1.005 \pm 0.67$ large \\
ProAgility $[\mathrm{s}]$ & $4.93 \pm 0.15$ & $4.93 \pm 0.087$ & $0.0 \pm 3.1$ & 1.000 & $0.008 \pm 1.02$ trivial \\
COD Deficit $[\mathrm{s}]$ & $-0.23 \pm 0.31$ & $0,20 \pm 0.11$ & & 0.034 & $1.013 \pm 0.61$ moderate \\
Bronco $[\mathrm{s}]$ & $279.13 \pm 5.62$ & $314.41 \pm 46.49$ & $11.8 \pm 10.1$ & 0.077 & $5.217 \pm 4.24$ very large \\
MAS $\left[\mathrm{m} \cdot \mathrm{s}^{-1}\right]$ & $4.28 \pm 0.10)$ & $3.89 \pm 0.43)$ & $-16.5 \pm 7.0$ & 0.026 & $-1.053 \pm 3.08$ very large \\
\hline
\end{tabular}

$\mathrm{d}$ : standardized effect size of Cohen; IMTP Avg = average isometric mid-thigh pull; CMJ = countermovement jump; DJ = drop jump; HJ = horizontal jump; $\mathrm{COD}=$ change of direction; $\mathrm{MAS}=$ maximal aerobic speed; $\mathrm{bw}=$ body weight .

changes to the analyzed period depended on each player's baseline fitness level, and the impact was greater among the baseline considered-fit soccer athletes.

During the pre-season, it is expected that soccer players will be exposed to greater loads because they need to compensate for drops in fitness levels that can occur during the off-season [2]. Therefore, respecting the principle of overload and training progression [28], it is expected that positive changes will occur in aerobic capacity, strength endurance, and repeated sprint ability [38]. However, considering the first objective of the present study, the athletes presented significant lower jump performance values and no significant changes in the overall variables after pre-season. The results of the present study contrast with other studies that have reported significant improvements in vertical jump performance after pre-season training $[6,40]$. In fact, a study conducted on 13 semiprofessional soccer players competing in England that analyzed seasonal fitness variations revealed significant improvements of aerobic, vertical jump and change of direction performance from the beginning to the end of the pre-season period. The decreases in jump performance 
Table 3. Unfit player changes between baseline and post assessments

\begin{tabular}{lccccc}
\hline Fitness determinants & Pre $($ mean $\pm \mathrm{SD})$ & Post $($ mean $\pm \mathrm{SD})$ & $\%$ change $\pm \mathrm{SD}$ (Post-Pre) & $\mathrm{p}$ & $\mathrm{d} \pm \mathrm{SD}$ \\
\hline IMTP Peak $[\mathrm{kg}]$ & $107.70 \pm 12.23$ & $109.34 \pm 24.79$ & $-0.1 \pm 10.2$ & 0.787 & $-0.007 \pm 0.81$ trivial \\
IMTP Avg $[\mathrm{kg}]$ & $96.55 \pm 10.85$ & $97.02 \pm 19.87$ & $-0.6 \pm 10.8$ & 0.932 & $-0.053 \pm 0.88$ trivial \\
Relative Force $[\mathrm{N} / \mathrm{kg}]$ & $13.14 \pm 1.93$ & $14.42 \pm 1.14$ & $10.5 \pm 20.4$ & 0.316 & $0.668 \pm 1.23$ moderate \\
CMJ $[\mathrm{cm}]$ & $31.85 \pm 2.04$ & $28.95 \pm 2.76$ & $-9.3 \pm 9.0$ & 0.102 & $-1.605 \pm 1.63$ large \\
DJ $[\mathrm{cm}]$ & $27.04 \pm 2.11$ & $27.24 \pm 1.39$ & $0.9 \pm 9.3$ & 0.866 & $0.122 \pm 1.24$ trivial \\
HJ $[\mathrm{cm}]$ & $221.88 \pm 10.37$ & $215.25 \pm 8.40$ & $-3.0 \pm 4.5$ & 0.249 & $-0.581 \pm 0.91$ small \\
ProAgility [s] & $5.29 \pm 0.15$ & $5.17 \pm 0.31$ & $-2.4 \pm 2.9$ & 0.181 & $-0.675 \pm 0.82$ moderate \\
COD Deficit [s] & $0.29 \pm 0.07$ & $0.23 \pm 0.09$ & & 0.288 & $-1.003 \pm 1.54$ moderate \\
Bronco [s] & $331.70 \pm 40.27$ & $325.84 \pm 27.30$ & $-1.4 \pm 9.2$ & 0.729 & $-0.132 \pm 0.85$ trivial \\
MAS [m $\left.\cdot \mathrm{s}^{-1}\right]$ & $3.48 \pm 0.36)$ & $3.62 \pm 0.32)$ & $4.2 \pm 14.3$ & 0.569 & $0.372 \pm 1.24$ small \\
\hline
\end{tabular}

d: standardized effect size of Cohen; IMTP Avg = average isometric mid-thigh pull; CMJ = countermovement jump; DJ = drop jump; HJ = horizontal jump; $\mathrm{COD}=$ change of direction; MAS = maximal aerobic speed;

after pre-season, found in the present study, can possibly be linked with neuromuscular fatigue factors due to the higher volume of training in this period. Or it could be that only soccer practice, in this soccer team, may not be enough for a better stretch-shortening cycle [24].

As mentioned earlier, improvements in aerobic capacity were reported at the end of the pre-season period $[7,21]$. Those improvements may be due to the higher training loads that are associated with greater volumes, with little variation observed in the training load variables [20], thus revealing higher values of training monotony and strain [10]. Therefore, and considering that in the present study we did not consider the training loads imposed on athletes during the pre-season, we only may suggest that the training principles were not properly followed. These discrepancies might be due to inadequate adjustments made during the pre-season training process or the number of games performed by players (which was also not controlled in the present study). Significant improvements were expected for all the analyzed variables, with greater emphasis on aerobic capacity due to the training process. Also, given the fact that amateur soccer players come from a longer off-season period than elite athletes, it is imperative to assess the athletes' physical fitness at the beginning of the season to adjust training in a manner that allows for positive changes over time.

Players with higher levels of physical performance (fit) at the baseline experienced decreases in overall physical performance during the pre-season period, whereas unfit players did not show any significant changes. The study conducted by Los Arcos et al. [18] showed that the players with the best baseline performances improved less than the players with the worst baseline performances in aerobic fitness. This is in line with our findings, although the present study revealed decreased physical performance values for fit players after the pre-season period. These decreases might be due to the need for more intense stimuli in order for changes to occur [27]. Therefore, these decreases might indicate a lack of individualization in the training program. Also, the players who started the preseason with lower physical performance values may have experienced physical improvements because the coaches and players failed to check whether fitness levels were maintained during the off-season, during which time a decrease in aerobic performance is expected [30]. The team assessed is from an amateur soccer league. Thus, there might be no planned activities for these players to perform during the off-season, and their off-season is longer than those of elite soccer teams. These factors could have also influenced the present findings.

This study had some limitations. One was related to the sample size, as we assessed only one soccer team; the assessment of another senior team under the same conditions and in the same context was not possible. Another limitation is the fact that only pre - and post-assessments for the pre-season period were considered. It would be interesting to analyze intra - and inter-week team variations in physical performance and load during the competition phase. It would also be worthwhile to examine within and between-variations data among different playing positions during the pre-season in amateur soccer teams, given the differences in the training approaches between amateur and elite leagues. Despite these limitations, to the best of our knowledge, this was the first study to investigate 
the variations in physical fitness determinants and to analyze Portuguese amateur soccer players' responses to the training process over the pre-season. Therefore, this study provides new insight into amateur football and presents several practical implications.

Amateur soccer teams might lack the conditions to monitor internal loads and, more specifically, external loads because these teams cannot afford external load monitoring equipment such as global positioning systems, which could be essential for the monitoring of training during the pre-season [9]. Fortunately, even amateur teams can afford to monitor internal loads via the subjective session-rate of perceived exertion (s-RPE) modified by Foster et al. [11]. From s-RPE, coaches can gather more training load data regarding the acute and chronic loads and their ratio (acute:chronic load), which prevents spikes in load, and reaching detraining and/or overreaching [11, 12]. Also, from the same subjective monitoring tool, coaches can analyze weekly training variability and tension from the calculation of training monotony and strain, respectively. Given that, even with low budgets, coaches can plan and adjust pre-season training programs with discretion that will improve players' performance.

\section{Conclusions}

The present study aimed to analyze the changes in physical variables among players from an amateur soccer team after the pre-season. The results revealed a lack of significant positive changes after the assessed period. Only the players considered fit at the baseline presented significant performance decreases. As amateur soccer players have a longer off-season period, the assessment of individual physical qualities at the beginning of preseason is imperative to allow coaches to plan and apply the necessary adjustments of training loads, and to track meaningful changes after this critical period. Also, even in amateur soccer contexts, coaches and athletes would benefit from the use of tools such as s-RPE for a better understanding of what is happening during the training process.

Conflict of interest: Authors state no conflict of interest.

\section{References}

1. Baker D. (2011) Recent trends in highintensity aerobic training for field sports. J. Strength Cond. Res., 22: 3-8.

2. Bangsbo J., Mohr M., Krustrup P. (2006) Physical and metabolic demands of training and match-play in the elite football player. J. Sports Sci., 24(7): 665-674. DOI: 10.1080/02640410500482529.
3. Bizati O. (2016) Physical and physiological characteristics of an Elite soccer team's players according to playing positions. Anthropologist., 26(3): 175-80. DOI: 10.1080/09720073.2016.11892146.

4. Blas X., Padullés J., Amo J., Balic M. (2007) Creation and Validation of Chronojump-Boscosystem: A Free Tool to Measure Vertical Jumps. Int. J. Sport. Sci., 30(8): 334356. DOI: 10.5232/ricyde2012.03004.

5. Boone J., Vaeyens R., Steyaert A., Bossche L., Bourgois J. (2012) Physical Fitness of Elite Belgian Soccer Players By Player Position. J. Strength Cond. Res./Natl. Strength Cond. Assoc., 26(8): 2051-257. DOI: 10.1519/ JSC.0b013e318239f84f.

6. Caldwell B., Peters D. (2009) Seasonal Variation in Physiological Fitness of a Semiprofessional Soccer Team. J. Strength Cond. Res., 23(5): 1370-77. DOI: 10.1519/ JSC.0b013e3181a4e82f.

7. Casajús J. (2001) Seasonal variation in fitness variables in professional soccer player. J. Sports Med. Phys. Fitness, 41(9): 463.

8. Clemente F.M., Owen A., Serra-Olivares J., Nikolaidis P.T., Van Der Linden C.M.I., Mendes B. (2019) Characterization of the Weekly External Load Profile of Professional Soccer Teams from Portugal and the Netherlands. J. Hum. Kinet., 66(1): 155-164. DOI: 10.2478/ hukin-2018-0054.

9. Clemente F.M., Seerden G., van der Linden C.M.I. (2019) Quantifying the physical loading of five weeks of preseason training in professional soccer teams from Dutch and Portuguese leagues. Physiol. Behav., 209: 112588. DOI: 10.1016/j.physbeh.2019.112588.

10. Clemente F.M., Silva R., Castillo D., Arcos A.L., Mendes B. (2020) Weekly Load Variations of DistanceBased Variables in Professional Soccer Players: A FullSeason Study. Int. J. Environ. Res. Public Health, 17(9): 3300. DOI: 10.3390/ijerph17093300.

11. Foster C. (1998) Monitoring training in athletes with reference to overtraining syndrome. Med. Sci. Sports Exerc., 30: 1164-1168. DOI: 10.1097/00005768-19980700000023.

12. Gabbett T.J. (2016) The training-injury prevention paradox: Should athletes be training smarter and harder? $B r$. J. Sports Med., 50(5): 273-80. DOI: 10.1136/bjsports2015-095788.

13. Harper D.J., Carling C., Kiely J. (2019) High-Intensity Acceleration and Deceleration Demands in Elite Team Sports Competitive Match Play: A Systematic Review and Meta-Analysis of Observational Studies. Sport. Med., 49(12): 1923-1947. DOI: 10.1007/s40279-019-01170-1.

14. Haugen T.A. (2018) Soccer seasonal variations in sprint mechanical properties and vertical jump performance. Kinesiology, 50: 102-108.

15. Kelly R.A., Collins K. (2018) The seasonal variations in anthropometric and performance characteristics of 
elite intercounty gaelic football players. J. Strength Cond. Res., 32(12): 3466-3473. DOI: 10.1519/ jsc.0000000000001861.

16. Kelly V., Wood A. (2013) The correlation between the 30-15 intermittent fitness test and a novel test of running performance. J. Aust. Strength Cond., 21(1): 91-94.

17. Leão C., Camões M., Clemente F.M., Nikolaidis P.T., Lima R., Bezerra P., Rosemann T., Knechtle B. (2019) Anthropometric Profile of Soccer Players as a Determinant of Position Specificity and Methodological Issues of Body Composition Estimation. Int. J. Environ. Res. Public Health, 16(13): 2386. DOI: 10.3390/ijerph16132386.

18. Los Arcos A., Castillo D., Martínez-Santos R. (2018) Influence of initial performance level and tactical position on the aerobic fitness in soccer players after preseason period. Sci. Med. Footb., 2(4): 294-298 DOI: 10.1080/24733938.2018.1451652.

19. Magal M., Smith R., Dyer J., Hoffman J. (2006) Seasonal Variation in Physical Performance-Related Variables in Male NCAA Division III Soccer Players. J. Strength Cond. Res./Natl. Strength Cond. Assoc., 20(4): 874-881. DOI: 10.1519/JSC.0b013e3181b3ddbf.

20. Malone J.J., Di Michele R., Morgans R., Burgess D., Morton J.P., Drust B. (2015) Seasonal Training-Load Quantification in Elite English Premier League Soccer Players. Int. J. Sports Physiol. Perform., 10(4): 489-497. DOI: 10.1123/ijspp.2014-0352.

21. Meckel Y., Doron O., Eliakim E., Eliakim A. (2018) Seasonal Variations in Physical Fitness and Performance Indices of Elite Soccer Players. Sports, 6(1): 14. DOI: 10.3390 /sports6010014.

22. Metaxas T., Sendelides T., Koutlianos N., Mandroukas K. (2006) Seasonal variation of aerobic performance in soccer players according to positional role. J. Sports Med. Phys. Fitness, 46(4): 520-525.

23. Mohr M., Krustrup P., Bangsbo J. (2003) Match performance of high-standard soccer players with special reference to development of fatigue. J. Sports Sci., 21(7): 519-528. DOI: 10.1080/0264041031000071182.

24. Nicol C., Avela J., Komi P.V. (2006) The stretch-shortening cycle: A model to study naturally occurring neuromuscular fatigue. Sport Med., 36(11): 977-99. DOI: 10.2165/00007256-200636110-00004.

25. Nimphius S., Callaghan S.J., Spiteri T., Lockie R.G. (2016) Change of Direction Deficit: A More Isolated Measure of Change of Direction Performance Than Total 505 Time. J. Strength Cond. Res., 30(11): 3024-32. DOI: 10.1519/JSC.0000000000001421.

26. Nygaard Falch H., Guldteig Rædergård H., van den Tillaar R. (2019) Effect of Different Physical Training Forms on Change of Direction Ability: a Systematic Review and Meta-analysis. Sports Med. - Open, 5(1): 1-37. DOI: 10.1186/s40798-019-0223-y.
27. Pickering C., Kiely J. (2019) Do Non-Responders to Exercise Exist - and If So, What Should We Do About Them? Sports Med., 49(1): 1-7. DOI: 10.1007/s40279018-01041-1.

28. Powers S., Howley E. (2014) Exercise Physiology: Theory and Application to Fitness and Performance. New York: Manole. 8th ed. ISBN: 978-85-204-3676-9.

29. Reilly T., George K., Marfell-Jones M., Scott M., Sutton L., Wallace J.A. (2009) How well do skinfold equations predict percent body fat in elite soccer players? Int. J. Sports Med., 30(8): 607-613. DOI: 10.1055/s-00291202353.

30. Requena B., García I., Suárez-Arrones L., Sáez De Villarreal E., Naranjo Orellana J., Santalla A. (2017) OffSeason Effects on Functional Performance, Body Composition, and Blood Parameters in Top-Level Professional Soccer Players. J. Strength Cond. Res., 31(4): 939-946. DOI: 10.1519/JSC.0000000000001568.

31. Ruivo R.M., Carita A.I., Pezarat-Correia P. (2016) Effects of a 16-week strength-training program on soccer players. Sci. Sport, 31(5): e107-13. DOI: 10.1016/j. scispo.2016.02.008.

32. Silva J.R., Nassis G.P., Rebelo A. (2015) Strength training in soccer with a specific focus on highly trained players. Sports Med. - Open, 1(1): 1-27 DOI: 10.1186/ s40798-015-0006-z.

33. Slimani M., Znazen H., Miarka B., Bragazzi N.L. (2019) Maximum Oxygen Uptake of Male Soccer Players According to their Competitive Level, Playing Position and Age Group: Implication from a Network Meta-Analysis. J. Hum. Kinet., 66(1): 233-245. DOI: 10.2478/hukin2018-0060.

34. Stewart P.F., Turner A.N., Miller S.C. (2014) Reliability, factorial validity, and interrelationships of five commonly used change of direction speed tests. Scand. J. Med. Sci. Sport, 24(3): 500-506. DOI: 10.1111/sms.12019.

35. Urquhart M., Bishop C., Turner A.N. (2018) Validation of a crane scale for the assessment of portable isometric mid-thigh pulls. J. Aust. Strength Cond., 25(5): 28-33. DOI: 10.13140/RG.2.2.26752.48642.

36. Wang R., Hoffman J.R., Tanigawa S., Miramonti A.A., La Monica M.B., Beyer K.S., Church D.D., Fukuda D.H., Stout J.R. (2016) Isometric Mid-Thigh Pull Correlates With Strength, Sprint, and Agility Performance in Collegiate Rugby Union Players. J. Strength Cond. Res., 30(11): 3051-3056. DOI: 10.1519/JSC.0000000000001416.

37. Wik E.H., Auliffe S.M., Read P.J. (2018) Examination of Physical Characteristics and Positional Differences in Professional Soccer Players in Qatar. Sports, 7(1): 9. DOI: $10.3390 /$ sports 7010009 .

38. Windt J., Gabbett T.J., Ferris D., Khan K.M. (2017) Training load-Injury paradox: Is greater preseason participation associated with lower in-season injury risk in 
elite rugby league players? Br. J. Sports Med., 51(8): 645-650. DOI: 10.1136/bjsports-2016-095973.

39. Wisløff U., Castagna C., Helgerud J., Jones R., Hoff J. (2004) Strong correlation of maximal squat strength with sprint performance and vertical jump height in elite soccer players. Br. J. Sports Med., 38(3): 285-288. DOI: 10.1136/bjsm.2002.002071.

40. Wong P-L., Chaouachi A., Chamari K., Dellal A., Wisløff U. (2010) Effect of Preseason Concurrent Muscular Strength and High-Intensity Interval Training in Professional Soccer Players. J. Strength Cond. Res., 24(3): 653-60. DOI: 10.1519/JSC.0b013e3181aa36a2.
Received 03.12.2020

Accepted 15.01.2021

(C) University of Physical Education, Warsaw, Poland

Acknowledgments

This study was done as part of a master thesis in sports training, Escola Superior de Desporto e Lazer, Instituto Politécnico de Viana do Castelo, Portugal. 